\title{
Inversão de Amplitudes para Caracterização e Monitoramento de Reservatórios
}

\author{
Petre Candelero Griscenco IAG/USP; Liliana Alcazar Diogo IAG/USP; Marcelo Belentani de Bianchi IAG/USP
}

Copyright 2014, SBGf - Sociedade Brasileira de Geofísica

Este texto foi preparado para a apresentação no VI Simpósio Brasileiro de Geofísica, Porto Alegre, 14 a 16 de outubro de 2014. Seu conteúdo foi revisado pelo Comitê Técnico do VI SimBGf, mas não necessariamente representa a opinião da SBGf ou de seus associados. É proibida a reprodução total ou parcial deste material para propósitos comerciais sem prévia autorização da SBGf.

\section{Resumo}

Este trabalho tem como objetivo implementar uma inversão sísmica quantitativa e não linear para investigar a relação entre as propriedades petrofísicas de um reservatório de hidrocarbonetos com os seus atributos sísmicos. Através de dados sísmicos sintéticos, gerados com configurações distintas de saturação pelo hidrocarboneto, investigou-se a viabilidade $e$ a sensibilidade do monitoramento do reservatório utilizando a sísmica.

\section{Introdução}

Tradicionalmente, na indústria de óleo e gás, a Geofísica sempre desempenhou papel fundamental na exploração de novos campos de hidrocarbonetos através do uso do método sísmico. Nas últimas décadas, entretanto, vem crescendo o uso do método sísmico na parte pósexploratória, no desenvolvimento dos campos, principalmente na caracterização e no monitoramento de campos já em fase de produção através da chamada "Sísmica 4D" ou "Sísmica time-lapse".

O método utilizado pela Sísmica 4D consiste em efetuar aquisições sísmicas (2D ou 3D) em diferentes momentos da vida útil do reservatório e comparar as aquisições buscando pelas diferenças entre elas. Deve-se, entretanto, buscar apenas as diferenças provenientes de variações petrofísicas dentro do reservatório e não diferenças decorrentes do fato de que as aquisições foram tomadas em momentos diferentes com condições diferentes. Para vencer esse problema são utilizadas diversas técnicas para aumentar a repetibilidade entre as aquisições sísmicas.

As primeiras inversões 4D apresentavam caráter qualitativo, eram utilizadas como uma ferramenta de auxílio no desenvolvimento dos campos. Nos últimos anos cada vez mais avança-se no sentido de tornar a sísmica 4D mais quantitativa e ela tem papel cada vez mais central no desenvolvimento dos campos (Grochau, 2013).

Diversos casos de sucesso do uso de sísmica 4D em dados reais já foram relatados (MacBeth et al., 2006; Thore \& Hubens, 2012; Veire, Borgos \& Landro, 2006), entretanto a grande maioria desses casos de sucesso ocorre em reservatórios areníticos menos rígidos e mais porosos, poucos são os casos de sucesso em reservatórios mais rígidos e menos porosos.

Um dos principais atributos sísmicos utilizado para interpretar as variações petrofísicas é a amplitude das reflexões. A maioria dos trabalhos empregam aproximações para as relações que descrevem a partição de energia do campo de onda nas interfaces (Aki \& Richards, 1980; Castagna, 1993). Essas aproximações são válidas para ângulos de incidência abaixo do ângulo crítico, região em que a sensibilidade é menor para variações das impedâncias das camadas. Ao se utilizar relações exatas que descrevem a partição da energia (equações de Zoeppritz), apesar de não-lineares e mais complicadas matematicamente, pode-se utilizar toda a faixa de ângulos disponíveis, principalmente ao redor do ângulo crítico, aonde a sensibilidade às variações de impedância é maior (Landrø, 2004). Para reservatórios mais rígidos e menos porosos, é usual a situação em que a impedância acústica do reservatório é maior do que a da rocha selante. Neste caso, existe ângulo crítico de incidência, Landrø (2004) verificou numericamente que pequenas variações da velocidade da onda $P$ geram variações significativas na distância crítica e propõem/avaliam utilizar a informação da variação da distância crítica para estimar variações de velocidade da rocha reservatório durante a produção.

Neste trabalho foi investigado o problema de inversão não linear das amplitudes refletidas no topo do reservatório. Foi considerada, inicialmente, uma situação simplificada para o modelo do reservatório para o desenvolvimento das análises da viabilidade $e$ sensibilidade do processo de inversão das amplitudes visando a extração dos parâmetros da rocha reservatório $\left\{V_{P}, V_{S}, \rho\right\}$.

\section{Metodologia e Problema Investigado}

O problema abordado consiste na simulação de um reservatório de hidrocarbonetos em produção e a partir de dois levantamentos sísmicos (também simulados numericamente) efetuados em tempos distintos da produção, estimar através da inversão as propriedades da rocha em cada um desses momentos. Monitorando, dessa forma, as variações com a produção do reservatório.

Em cada um dos momentos da produção, as amplitudes sísmicas foram simuladas, considerando-se os valores de velocidades e de densidade que caracterizam o estado do reservatório naquele momento. Utilizou-se a teoria de substituição de fluido de Gassmann (1951) que conecta as características petrofísicas do reservatório com a velocidade da onda sísmica propagando-se nele. 
A partir dos valores das velocidades $\left(V_{P} e V_{S}\right) \mathrm{e}$ densidade $(\rho)$ das rochas selante e reservatório foram gerados dados sintéticos para os coeficientes de reflexão da onda $\mathrm{P}$ no topo do reservatório em função do ângulo de reflexão, simulando assim, os levantamentos sísmicos. Para a geração desses dados foi utilizada a implementação exata das equações de Zoeppritz para as reflexões $\mathrm{PP}$, como escritas por Ikelle and Amundsen (2005), em função dos parâmetros $\left(V_{P}, V_{S} e \rho\right)$ dos meios acima e abaixo da interface refletora.

Para a inversão, a rocha selante foi assumida como conhecida e as incógnitas do problema foram os valores de $V_{P}, V_{S}$ e $\rho$ da rocha reservatório.

O algoritmo de inversão consistiu na solução do problema de otimização da Função Objetivo, definida como sendo a soma dos quadrados das diferenças entre os atributos observados (dados sintéticos) e os calculados. O atributo considerado foi a amplitude refletida no topo do reservatório.

O processo de inversão foi implementado em Matlab. Para a minimização da Função Objetivo, adaptou-se o algoritmo genético incluso nas bibliotecas do pacote. Algoritmos estocásticos de otimização aplicados em problemas não lineares raramente fornecem a mesma solução quando executados mais do que uma vez. O algoritmo genético foi executado 100 vezes e cada execução foi denominada de iteração do processo de inversão.

Em um primeiro momento foram abordadas análises referentes à performance do algoritmo de inversão em diversos cenários de porosidade da rocha reservatório. Em seguida o algoritmo de inversão foi aplicado em diferentes cenários de variação da saturação de óleo para verificar sua eficiência para o monitoramento do reservatório, verificando até que ponto é possível recuperar os valores corretos de velocidade e consequentemente de saturação de óleo

O reservatório simulado foi um arenito relativamente limpo de matriz mineral de quartzo com apenas duas fases: óleo e água. Os parâmetros que definem o modelo do reservatório encontram-se na Tabela 1. Como rocha selante, considerou-se um folhelho com as velocidades e densidades apresentadas na Tabela 2. Supôs-se que as camadas acima da rocha reservatório permaneceram constantes durante a produção.

As Figuras 1 e 2 mostram como a velocidade da onda $P$ e a densidade variam com a saturação para alguns valores de porosidade da rocha reservatório. Nota-se que quanto menor é o valor da porosidade, maior é a dificuldade para identificar variações no valor da velocidade e densidade dentro do reservatório. Esse resultado é condizente com o fato de que o uso de sísmica 4D apresenta maior sucesso em reservatórios mais porosos, ou seja, aonde a variação de velocidade é mais pronunciada para determinada variação de saturação.

\begin{tabular}{c|c}
\hline Parâmetro & Valor \\
\hline$K_{0}$ & $36.4 \mathrm{GPa}$ \\
$K_{H 2 O}$ & $2.95 \mathrm{GPa}$ \\
$K_{\text {oil }}$ & $1.05 \mathrm{GPa}$ \\
$\mu$ & $4 \mathrm{GPa}$ \\
$\rho_{0}$ & $2.65 \mathrm{~g} / \mathrm{cm}^{3}$ \\
$\rho_{H 2 O}$ & $1.04 \mathrm{~g} / \mathrm{cm}^{3}$ \\
$\rho_{\text {oil }}$ & $0.715 \mathrm{~g} / \mathrm{cm}^{3}$ \\
\hline Tabela $1-$ Parâmetros & Petrofísicos $\mathrm{do} \mathrm{Modelo} \mathrm{de}$ \\
Reservatório & \\
\hline$V_{P}$ & $2.8 \mathrm{~km} / \mathrm{s}$ \\
$V_{S}$ & $1.5 \mathrm{~km} / \mathrm{s}$ \\
$\rho$ & $2.3 \mathrm{~g} / \mathrm{cm}^{3}$ \\
\hline
\end{tabular}

Tabela 2 - Parâmetros do Folhelho Selante

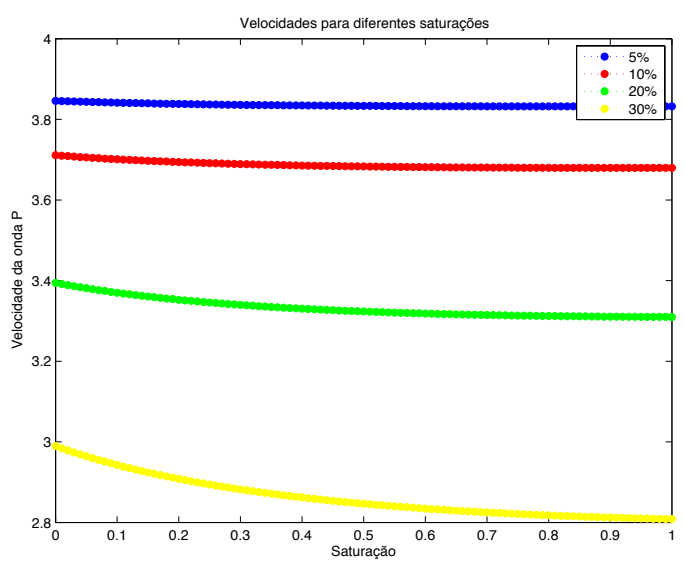

Figura 1 -Velocidade da onda $P$ para diferentes saturações e porosidades.

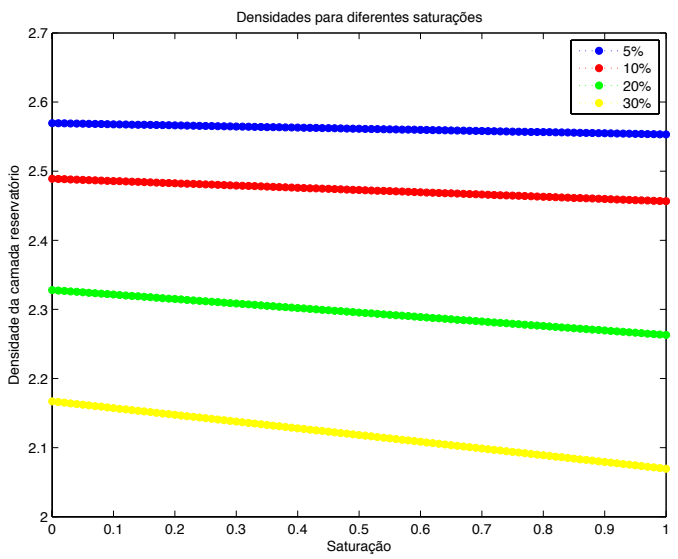

Figura 2 - Densidade do reservatório para diferentes saturações e porosidades. 

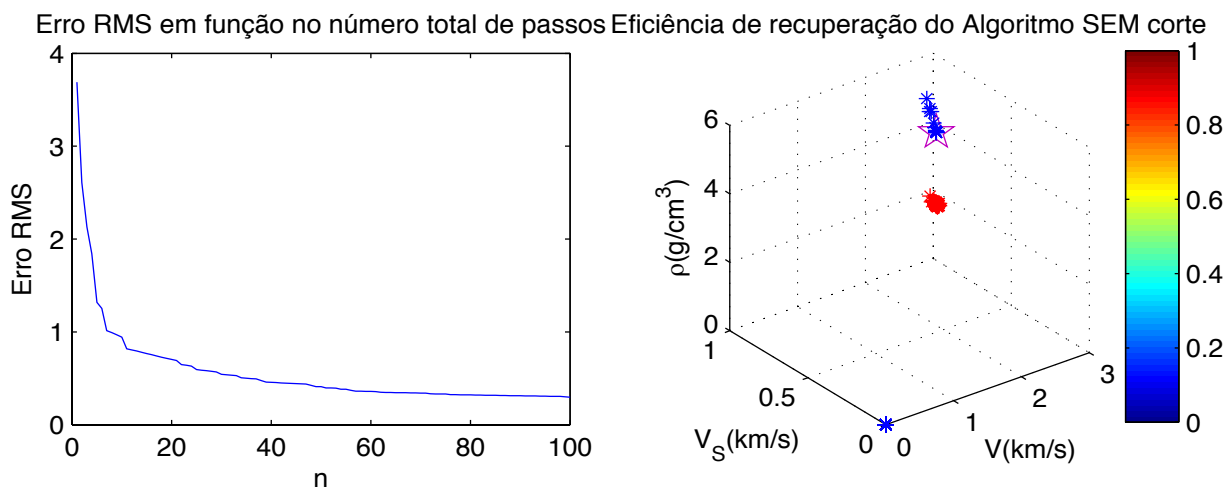

Eficiência de recuperação do Algoritmo COM corte

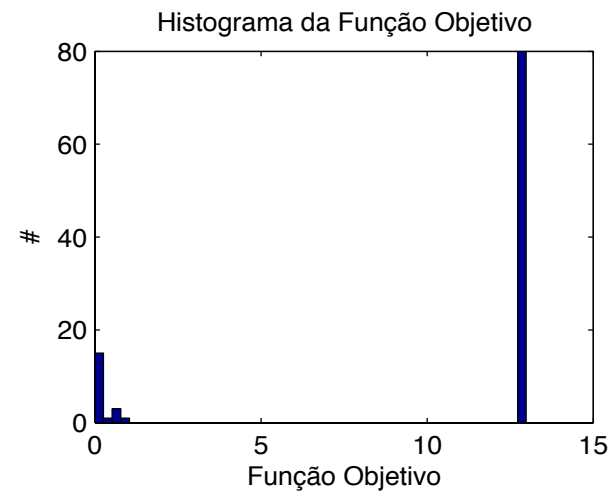

Figura 3 - Performance do algoritmo de inversão para rocha com porosidade $\phi=10 \%$. Ver descrição dos gráficos no texto.
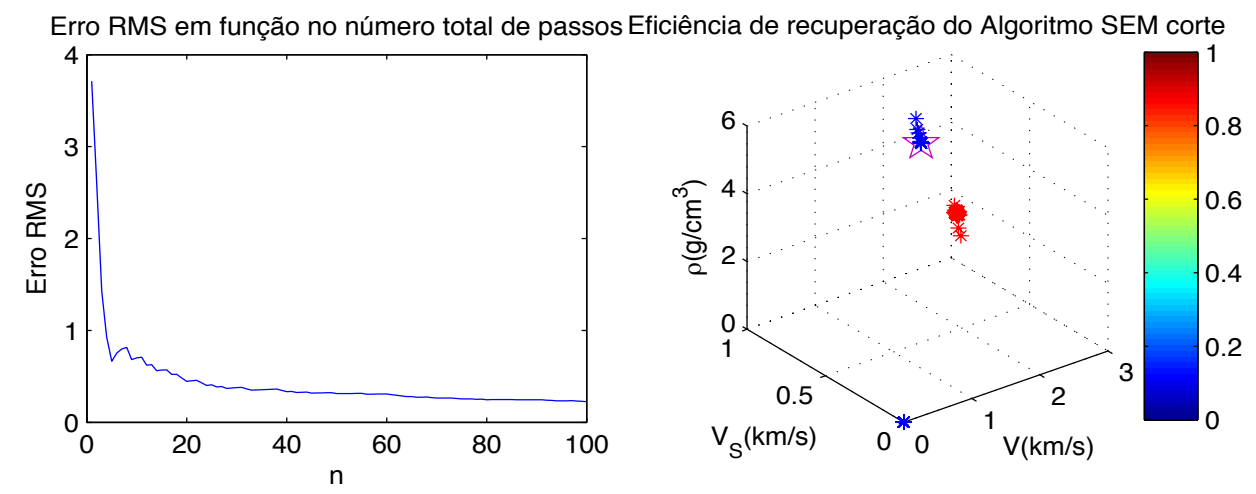

Eficiência de recuperação do Algoritmo COM corte

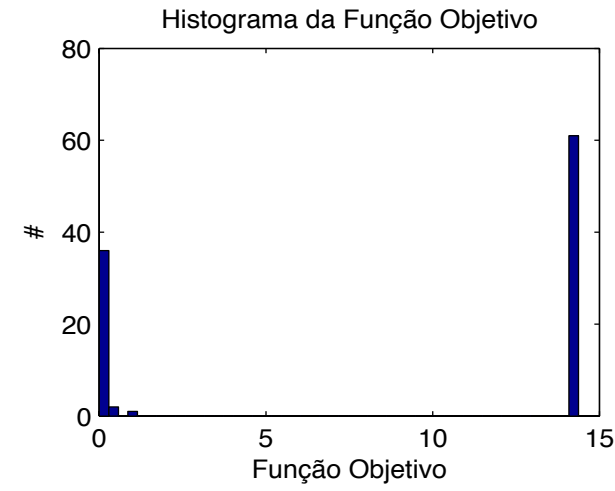

Figura 4 -Performance do algoritmo de inversão para rocha com porosidade $\phi=20 \%$. Ver descrição dos gráficos no texto. 


\section{Resultados}

Para a análise da performance do algoritmo de inversão, foram averiguadas as seguintes informações avaliadas sobre as soluções obtidas de cada iteração: i) como o RMS dos erros entre o valor real dos parâmetros do modelo e os valores efetivamente recuperados com a inversão varia conforme cresce o número de iterações; ii) a distribuição das soluções finais do algoritmo genético, quando executado várias vezes e iii ) o histograma de valores da função objetivo no ponto final de cada busca.

Nas figuras 3 e 4 , os resultados obtidos dos critérios citados acima, para modelos de rocha reservatório com porosidades de $10 \%$ e $20 \%$, respectivamente, são apresentados em quatro gráficos, conforme descrição e comentários a seguir.

O gráfico superior a esquerda, mostra a curva de variação do RMS dos erros pelo número de iterações $(n)$. Conforme o número de iterações do algoritmo aumenta, o erro cai. Nas 10 primeiras iterações o erro cai rapidamente, na sequência as soluções melhoram cada vez mais lentamente até permanecer aproximadamente constante a partir de 80 execuções do algoritmo genético.

O segundo gráfico, superior a direita, apresenta o espaço dos 3 parâmetros da inversão $\left\{V_{P}, V_{S} e \rho\right\}$, com os pontos marcando os valores recuperados em cada iteração. $O$ ponto indicado pela estrela vermelha corresponde aos valores corretos, ou seja, a solução esperada. A escala de cores representa $o$ valor da função objetivo normalizado pelo máximo valor dentro do conjunto de pontos representados.

O histograma de ocorrência dos valores da função objetivo é apresentado no gráfico inferior à direita. Observa-se a presença de dois picos principais onde se concentram os valores da função objetivo. Elas indicam que um número menor de soluções atingiu o mínimo global, e a forte presença de um mínimo local da função objetivo.

No gráfico inferior à esquerda são apresentadas apenas as soluções para os valores da função objetivo abaixo de um valor estabelecido como critério de corte. Observa-se, nesse caso, que todas as soluções selecionadas são muito próximas dos valores desejados.

Comparando-se os resultados descritos acima para as rochas com porosidade de 10\% (Figura 3) e 20\% (Figura 4), observa-se que para a rocha com maior porosidade, o RMS do erro cai de maneira mais rápida e a quantidade de resultados apresentando maior valor da função objetivo é menor. O que corrobora o fato das inversões realizadas para dados de rocha reservatório de maior de porosidade apresentarem maior eficiência.

Em seguida foram efetuados testes de sensibilidade para a recuperação das velocidades do reservatório e consequente recuperação do valor da saturação de óleo no reservatório. Na Tabela 3 estão os resultados para a inversão dos dados gerados para o modelo com porosidade de $10 \%$ e $20 \%$.
O próximo teste foi adicionar ruído aos dados, simulando possíveis erros de extração dos atributos. Na Figura 5 são ilustrados os coeficientes de reflexão com e sem ruído. Na Tabela 4 estão os resultados para a inversão dos dados adicionados de ruído, também considerandose as duas porosidades.

Observando-se os resultados, nota-se que para o reservatório mais poroso, a sensibilidade é ligeiramente maior na capacidade de recuperar os valores de saturação nos casos sem adição de ruídos tanto para baixa $(9,09 \%)$ quanto para alta $(50 \%)$ variação de saturação no reservatório.

Ao se adicionar ruído aos dados vemos que para a porosidade maior, o algoritmo é muito mais sensível na recuperação dos valores para as duas variações de saturação avaliadas.

\begin{tabular}{|c|c|c|}
\hline \multicolumn{3}{|c|}{ Porosidade de $10 \%$. Dados sem ruído } \\
\hline $\begin{array}{c}\text { Variação percentual } \\
\text { de }\end{array}$ & \multicolumn{2}{|c|}{ Valores } \\
\hline $\begin{array}{l}\text { Saturação no } \\
\text { reservatório }\end{array}$ & $9,09 \%$ & $50,00 \%$ \\
\hline Saturação recuperada & $8,97 \%$ & $49,89 \%$ \\
\hline $\begin{array}{l}\text { Velocidade no } \\
\text { reservatório }\end{array}$ & $\begin{array}{c}0,012 \% \\
\left(0,4 \cdot 10^{-3} \mathrm{~km} / \mathrm{s}\right)\end{array}$ & $\begin{array}{c}0,18 \% \\
\left(6,8.10^{-3} \mathrm{~km} / \mathrm{s}\right)\end{array}$ \\
\hline $\begin{array}{l}\text { Velocidade } \\
\text { recuperada }\end{array}$ & $\begin{array}{c}0,011 \% \\
\left(0,4 \cdot 10^{-3} \mathrm{~km} / \mathrm{s}\right)\end{array}$ & $\begin{array}{c}0,18 \% \\
\left(6,8.10^{-3} \mathrm{~km} / \mathrm{s}\right)\end{array}$ \\
\hline \multicolumn{3}{|c|}{ Porosidade de $20 \%$. Dados sem ruído } \\
\hline $\begin{array}{c}\text { Variação percentual } \\
\text { de }\end{array}$ & \multicolumn{2}{|c|}{ Valores } \\
\hline $\begin{array}{l}\text { Saturação no } \\
\text { reservatório }\end{array}$ & $9,09 \%$ & $50,00 \%$ \\
\hline Saturação recuperada & $9,06 \%$ & $50,01 \%$ \\
\hline $\begin{array}{l}\text { Velocidade no } \\
\text { reservatório }\end{array}$ & $\begin{array}{c}0,055 \% \\
\left(1,8 \cdot 10^{-3} \mathrm{~km} / \mathrm{s}\right) \\
\end{array}$ & $\begin{array}{c}0,600 \% \\
\left(20.10^{-3} \mathrm{~km} / \mathrm{s}\right) \\
\end{array}$ \\
\hline $\begin{array}{l}\text { Velocidade } \\
\text { recuperada }\end{array}$ & $\begin{array}{c}0,055 \% \\
\left(1,8 \cdot 10^{-3} \mathrm{~km} / \mathrm{s}\right)\end{array}$ & $\begin{array}{c}0,600 \% \\
\left(20.10^{-3} \mathrm{~km} / \mathrm{s}\right)\end{array}$ \\
\hline
\end{tabular}

Tabela 3 - Sensibilidade do algoritmo para dados sem ruído. 


\begin{tabular}{|c|c|c|}
\hline \multicolumn{3}{|c|}{ Porosidade de $10 \%$. Dados com ruído } \\
\hline $\begin{array}{c}\text { Variação percentual } \\
\text { de }\end{array}$ & \multicolumn{2}{|c|}{ Valores } \\
\hline $\begin{array}{l}\text { Saturação no } \\
\text { reservatório }\end{array}$ & $9,09 \%$ & $50,00 \%$ \\
\hline Saturação recuperada & $10,80 \%$ & $55,41 \%$ \\
\hline $\begin{array}{l}\text { Velocidade no } \\
\text { reservatório }\end{array}$ & $\begin{array}{c}0,012 \% \\
\left(0,4 \cdot 10^{-3} \mathrm{~km} / \mathrm{s}\right)\end{array}$ & $\begin{array}{c}0,18 \% \\
\left(6,8.10^{-3} \mathrm{~km} / \mathrm{s}\right)\end{array}$ \\
\hline $\begin{array}{l}\text { Velocidade } \\
\text { recuperada }\end{array}$ & $\begin{array}{c}0,023 \% \\
\left(0,8 \cdot 10^{-3} \mathrm{~km} / \mathrm{s}\right)\end{array}$ & $\begin{array}{c}0,25 \% \\
\left(9,3 \cdot 10^{-3} \mathrm{~km} / \mathrm{s}\right)\end{array}$ \\
\hline \multicolumn{3}{|c|}{ Porosidade de $20 \%$. Dados com ruído } \\
\hline $\begin{array}{c}\text { Variação percentual } \\
\text { de }\end{array}$ & \multicolumn{2}{|c|}{ Valores } \\
\hline $\begin{array}{l}\text { Saturação no } \\
\text { reservatório }\end{array}$ & $9,09 \%$ & $50,00 \%$ \\
\hline Saturação recuperada & $9,04 \%$ & $50,34 \%$ \\
\hline $\begin{array}{c}\text { Velocidade no } \\
\text { reservatório }\end{array}$ & $\begin{array}{c}0,055 \% \\
\left(1,8.10^{-3} \mathrm{~km} / \mathrm{s}\right) \\
\end{array}$ & $\begin{array}{c}0,600 \% \\
\left(20.10^{-3} \mathrm{~km} / \mathrm{s}\right) \\
\end{array}$ \\
\hline $\begin{array}{l}\text { Velocidade } \\
\text { recuperada }\end{array}$ & $\begin{array}{c}0,055 \% \\
\left(1,8.10^{-3} \mathrm{~km} / \mathrm{s}\right)\end{array}$ & $\begin{array}{c}0,620 \% \\
\left(20.10^{-3} \mathrm{~km} / \mathrm{s}\right)\end{array}$ \\
\hline
\end{tabular}

Tabela 4 - Sensibilidade do algoritmo para dados com adição de $7 \%$ de ruído.

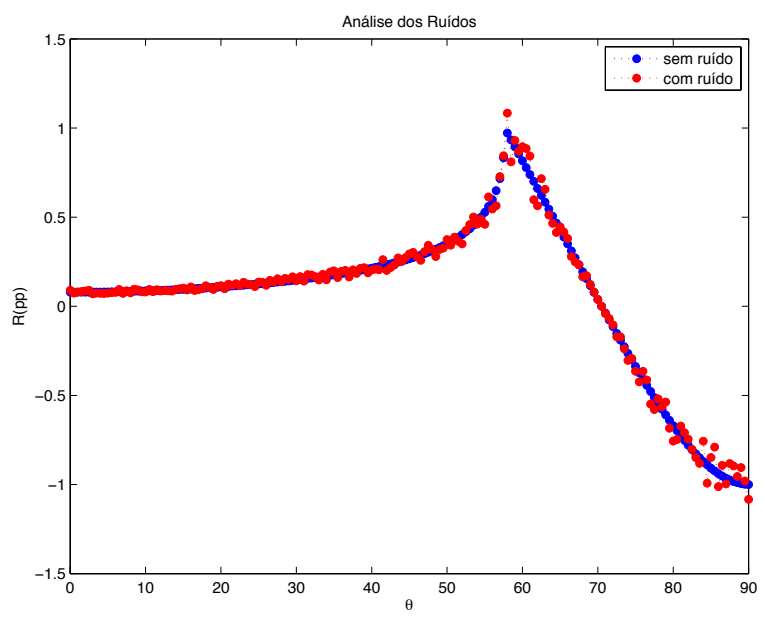

Figura 5 - Dados do coeficiente de reflexão com e sem ruído.

\section{Discussão e Conclusões}

Nota-se, de maneira geral, um melhor desempenho do algoritmo de inversão no reservatório mais poroso tanto na sua performance quanto na sua sensibilidade para a recuperação dos valores de saturação com o intuito de monitorar a produção do reservatório.
Ainda assim, mostrou-se que existe sensibilidade numérica do algoritmo de inversão para modelos de porosidade mais baixa $(10 \%)$ e foi possível conseguir recuperar de maneira satisfatória as variações de saturação e velocidades, embora com um erro maior na estimativa desses valores.

Entretanto, em dados reais, as variações de amplitude entre os levantamentos podem não ser suficientes para serem detectadas. Ou seja, pode não haver sensibilidade para se observar as variações de amplitude, devido a problemas de repetibilidade (ruído 4D).

Neste estudo, para a porosidade $\phi=10 \%$ e variação de saturação de $50 \%$ a variação percentual das amplitudes foi em média abaixo de $2 \%$ atingindo $5 \%$ na distância crítica. Já para a porosidade $\phi=20 \%$ com a mesma variação de saturação, as variações de amplitude são acima de $5 \%$ atingindo $20 \%$ na distância crítica. Portanto, provavelmente, para a porosidade menor não seria possível a aplicação do método para a recuperação das velocidades e saturações utilizando dados reais.

Recomenda-se para melhorar o processo de inversão, incorporar novos atributos sísmicos, como os tempos de chegada e o valor da distância crítica, no algoritmo com o intuito de se obter maior robustez na inversão e no monitoramento elevando dessa forma a capacidade de distinguir pequenas variações de saturação em reservatórios e variações em reservatório pouco porosos.

\section{Referências}

BERRYMAN, J. G. Tutorial Origin of Gassmann 's equations. Geophysics, v. 5, n. 5, p. 1627-1629, 1999.

CASTAGNA, J.; SWAN, H. Principles of AVO crossplotting - Shuey's Two-Term Approximation. The Leading Edge, v. 2, 1997.

GROCHAU, M. Time-Lapse (4D) Reservoir Monitoring. 13th International Congress of the SBGf, v. 1, p. 1-44, 2013.

HAN, D.; BATZLE, M. L. Gassmann's equation and fluidsaturation effects on seismic velocities. Geophysics, v. 69 , n. 2, p. 398-405, 2004.

HAN, D.; NUR, A.; MORGAN, D. Effects of porosity and clay content on wave velocities in sandstones. Geophysics, v. 51, n. 11, p. 2093-2107, 1986.

LANDR $\varnothing$, M.; NGUYEN, A. K.; MEHDIZADEH, H. Time lapse refraction seismic - a tool for monitoring carbonate fields? SEG Int 'I Exposition and 74th Annual Meeting Denver, Colorado, v. 2, p. 3-6, 2004.

LANDR $\varnothing, M$. Future challenges and unexplored methods for 4D seismic analysis. CSEG Recorder, p. 128-135, 2006. 
LANDR $\varnothing$, M.; STAMMEIJER, J. Quantitative estimation of compaction and velocity changes using 4D impedance and traveltime changes. Geophysics, v. 69, n. 4, p. 949957, 2004.

MACBETH, C.; FLORICICH, M.; SOLDO, J. Going quantitative with 4D seismic analysis. Geophysical Prospecting, p. 303-317, 2006.

NUR, A. et al. Critical porosity: A key to relating physical properties to porosity in rocks. The Leading Edge, p. 878881, 1998.

RUSSELL, B. H. et al. Fluid-property discrimination with AVO : A Biot-Gassmann perspective. Geophysics, v. 68, n. 1, p. 29-39, 2003.
THORE, P.; HUBANS, C. 4D seismic-to-well tying, a key step towards 4D inversion. Geophysics, v. 77, n. 6, p. R227-R238, 2012.

VEIRE, H. H.; BORGOS, H. G.; LANDR $\varnothing$, M. Stochastic inversion of pressure and saturation changes from timelapse AVO data. Geophysics, v. 71, n. 5, p. C81-C92, set. 2006.

WANG, Z. Fundamentals of seismic rock physics. Geophysics, v. 66, n. 2, p. 398-412, 2001. 